\title{
Sliding Charge Density Wave Induces Electron Transport
}

\author{
P. Monceau and M. Renard, Grenoble
}

(Centre de Recherches sur les Très Basses Températures)

Over the past fifteen years, intensive experimental and theoretical work has been undertaken to understand the physical properties of systems with restricted dimensionality. A low-dimensional conductor has an electrical conductivity that is strongly anisotropic with respect to the crystal structure - a specimen made up of infinite parallel chains for example, will exhibit a high conductivity only in the chain direction. Interest in this type of phenomenon has been greatly stimulated by the recently-acquired possibilities of synthesizing new families of inorganic as well as organic low-dimensional conductors. In a great number of these compounds the interaction between ions and electrons, the so-called electron-phonon interaction, can cause a modulated collective deformation of the electronic charge density to give a lower energy state at low temperature, as was first pointed out by Peierls ${ }^{1}$ ).

Peierls Transition and Fröhlich Conductivity

As is well known from band theory, every Brillouin zone constitutes a locus of discontinuity for the electronic energy. If, in a one-dimensional electronic system with a Fermi vector of $k_{F}$, a periodic lattice distortion of wave-vector $2 k_{\mathrm{F}}$ is introduced, the band structure will be modified because of the new periodicity. A new Brillouin zone appears at $\left|k_{\mathrm{F}}\right|$ and so, each occupied electronic energy for $|k|<k_{\mathrm{F}}$ decreases, giving rise to a new ground state of the system characterized by a charge density wave (CDW) with wave vector $q=2 k_{\mathrm{F}}$. The occupied electronic states are Bloch wave functions with the superlattice periodicity:

$$
\psi_{k}=\exp (i k r) \sum_{n} V_{k, n} \exp (\mathrm{i} n q r)
$$

and consequently the electronic density has Fourier components with wave-vectors $\pm n q$, especially for the fundamental ones $\pm q$ :

$\rho_{\mathrm{el}}=\rho_{\mathrm{o}}+2 \rho_{q} \cos (q r+\phi)+\ldots$ (1) where $\rho_{0}$ is the uniform electron density and $2 \rho_{q}$ the charge modulation amplitude. The phase, $\phi$, specifies the position of the CDW relative to the lattice ions. The local electron charge density is partially neutralized by a concomitant displacement of each ion to a new equilibrium position, the displacement of the $n^{\text {th }}$ ion, initially at $n r_{0}$, being

$$
u_{n}=u_{0} \sin \left(n q r_{0}+\phi\right)
$$

Since a gap, $\triangle$, is opened at the Fermi level, the CDW state has an energy lower than the metallic state.

CDW formation has also been observed in two-dimensional layered compounds, namely transition metal dichalcogenides, the Fermi surface of which approximates to a cylinder with nearly parallel faces. Thus a large fraction of states on the Fermi surface are connected by the same vector $q=2 k_{F}$ (the nesting condition). Nevertheless, the low-temperature ground state remains metallic. In contrast, for a strictly onedimensional conductor, the Fermi surface consists of two parallel planes, so that all states are connected by the same $q$. The energy gap removes the whole Fermi surface, and the low temperature ground state is insulating.

In the strictly one-dimensional case no long range order can be established because of fluctuations and there is no phase transition at any temperature. In practice, however, with pseudo onedimensional conductors, we can identify a characteristic temperature, called the Peierls transition temperature below which a lattice distortion occurs and the condensed state can be described by an order parameter. The latter can be defined either in terms of the electron density modulation as $\rho_{q} \exp (\mathrm{i} \phi$ ) (see equation 1) or in terms of the lattice distortion which is proportional to $\rho_{q}$.

Modulation of the ion positions can be detected by X-ray, neutron or electron diffraction measurements: superlattice spots appear near the main Bragg spots that correspond to the unmodulated structure. Measurements of the inverse separation of these superlattice spots give the CDW wave length. In real space, images of CDWs have been obtained using high resolution electronic diffraction. This method is very well suited to study defects in the CDW lattice induced, for instance, by electron irradiation.

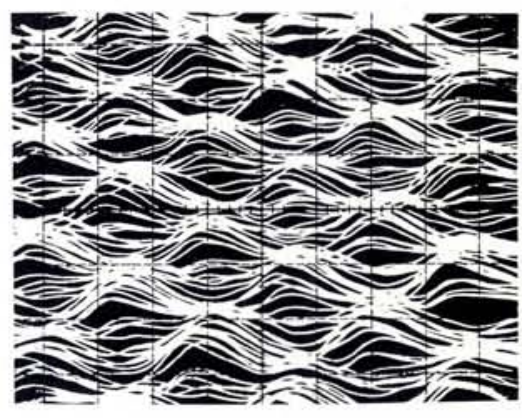

\section{$\rightarrow 11 \mathrm{nmk}$}

Fig. 1 - Charge density waves on a surface of $1 \mathrm{TTTS}{ }_{2}$ below the Peierls transition detected by a tunnelling microscope. The spacing between the mounds which form a hexagonal array is the CDW wavelength (from reference 2).

Very recently the use of a scanning tunnelling microscope on a cleaved surface of the two-dimensional $1 \mathrm{TTaS}_{2} \mathrm{CDW}$ compound has revealed that the real space CDW structure is formed by hexagonal arrays of mounds with the CDW wavelength spacing ${ }^{2}$ ) (Fig. 1).

The opening of a gap below the Peierls transition temperature is reminiscent of semiconductors, but the essential feature of a CDW is that its wavelength, $\lambda_{\text {CDW }}=2 \pi / 2 k_{F}$, is controlled by the Fermi surface dimensions and is generally unrelated to the undistorted lattice periodicities, i.e. the CDW is incommensurate with the lattice. Consequently the crystal no longer has a translation group and in contrast to semiconductors, the phase, $\phi$, of the lattice distortion is not fixed relative to the lattice but is able to slide along $q$. This phenomenon is easy to understand if we recognize that if the lattice is regular, no position is energetically favoured and no locking results. In more theoretical terms: if we think of the CDW as resulting from an electronic interaction via the lattice phonons, this interaction is the same in every galilean frame, provided that the frame velocity is small compared with the sound velocity (in which case the interaction would be strongly modified). CDW condensation may thus arise in any set of galilean frames with uniform velocity, $v$, giving in the laboratory frame an electronic current density,

$$
J=-n_{\mathrm{o}} e \mathrm{v}
$$

where $n_{0}$ is of the order of the electron number density condensed in the band below the CDW gap.

This model of a sliding CDW was proposed by Fröhlich in $1954^{3}$ ) as a mechanism which could lead to a superconducting state. This Fröhlich mode is a direct consequence of translation inva- 
riance. In practice, as shown by Lee, Rice and Anderson ${ }^{4}$ ) this translation invariance is broken because the phase, $\phi$, can in fact be pinned to the lattice, for example by impurities or by a longperiod commensurability between the CDW wavelength and the lattice. Oscillations of the pinned CDW are expected to produce a large low-frequency $A C$ conductivity and a large dielectric constant. An applied DC electric field, however, can supply the CDW with an energy sufficient to overcome the pinning, so that above a threshold field, the CDW can slide and carry a current. Damping prevents superconductivity. This extra conductivity associated with the collective CDW motion, called Fröhlich conductivity, has recently been observed ${ }^{5}$ ).

\section{Materials}

Up to now three families of inorganic compounds have been found to exhibit non-linear transport properties at temperatures below the Peierls transition, namely: the transition metal trichalcogenides such as $\mathrm{NbSe}_{3}, \mathrm{NbS}_{3}, \mathrm{TaS}_{3}$ with monoclinic or orthorhombic structures; the molybdenum oxides

$\mathrm{K}_{0.30} \mathrm{MoO}_{3}$ and $\mathrm{Rb}_{0.30} \mathrm{MoO}_{3}$ called blue bronzes and halogened transition metal tetrachalcogenides such as $\left(\mathrm{NbSe}_{4}\right)_{2} \mathrm{l}$, $\left(\mathrm{NbSe}_{4}\right)_{10} \mathrm{I}_{3},\left(\mathrm{TaSe}_{4}\right)_{2} \mathrm{I}$. Without going into detail, the structure of these compounds can be described as comprising: chains of trigonal prisms stacked on top of each other with a cross-section close to an isosceles triangle in the case of $\mathrm{NbSe}_{3}$, layers in the case of $\mathrm{K}_{0.3} \mathrm{MoO}_{3}$, and parallel $\left(\mathrm{TaSe}_{4}\right)$ chains with iodine atoms lying between them in the case of $\left(\mathrm{TaSe}_{4}\right)_{2} \mathrm{l}$. Whilst $\mathrm{NbSe}_{3}$ remains metallic at low temperature, all the other compounds exhibit a semiconducting behaviour below the Peierls transition temperature which, depending on the compound, lies between $330 \mathrm{~K}$ (for $\mathrm{NbS}_{3}$ ) and $59 \mathrm{~K}$ (for $\mathrm{NbSe}_{3}$ ). The wavelength of the CDW distortion appears to be incommensurate, but is very often near four lattice distances along the chain direction. A temperature dependence of the CDW wavelength has only been detected in orthorhombic $\mathrm{TaS}_{3}$ and blue bronze with an apparent commensurability setting at low temperature.

\section{Properties of the CDW Current-carrying State}

Since the first observation in $1976^{6}$ ) of non-linear transport properties when a DC or a microwave field was applied to $\mathrm{NbSe}_{3}$, the properties of this new current-carrying state have been widely studied; they can be summarized as
Fig. 2 - Variation of the nonlinear electrical conductivity (normalized to the Ohmic value) as a function of the reduced electric field for an orthorhombic $\mathrm{TaS}_{3}$ sample. The insert shows the V(I) characteristics.

follows:

- The DC electrical conductivity increases above a threshold field $E_{\mathrm{T}}$.

- The conductivity is strongly frequency-dependent in the range of $100 \mathrm{MHz}$ few $\mathrm{GHz}$.

- Above the threshold field, a timedependent voltage is generated in the crystal which can be analysed as the combination of a periodic component and a broad band noise following a $1 / f$ variation.

- Interference effects occur between the $A C$ voltage generated in the crystal in the non-linear state and an external RF field.

- Hysteresis and memory effects are observed, principally at low temperature.

Fig. 2 shows the typical variation of the electrical conductivity $\sigma$ (normalized to the ohmic value) as a function of electric field. The corresponding $V(I)$ characteristic is drawn in the inset; a deviation from Ohm's law is observed above a critical current $I_{\top}$ which leads to a threshold field defined as $E_{\mathrm{T}}=R I_{\mathrm{T}} / \ell$ where $R$ is the resistance of the sample and $\ell$ the distance between voltage contacts.

$E_{\mathrm{T}}$ varies typically froma few $\mathrm{mV} / \mathrm{cm}$ in $\mathrm{NbSe}_{3}$ to a few tenths of a V/cm in the other compounds; moreover $E_{\mathrm{T}}$ increases strongly when $T$ is lowered. A number of phenomenological laws have been derived to fit the conductivity variation $\sigma(E)$, amongst which one that has been widely used is reminiscent of a kind of interband tunnelling process, originally envisaged by Zener:

$\sigma(E)=\sigma_{a}+\sigma_{b}\left[1-E_{\mathrm{T}} / E\right] \exp \left(-E_{0} / E\right)$ where $\sigma_{a}$ is the ohmic conductivity and $E_{0}=C E_{\mathrm{T}}$ with $C$ between 2 and 5 . Such a low activation field $E_{0}$ precludes a single electron process because the bandgap which can be derived from Zener's theory is several orders of magnitude smaller than the thermal energy $k_{\mathrm{B}} T$. When $E \gg E_{\mathrm{T}}$ the conductivity

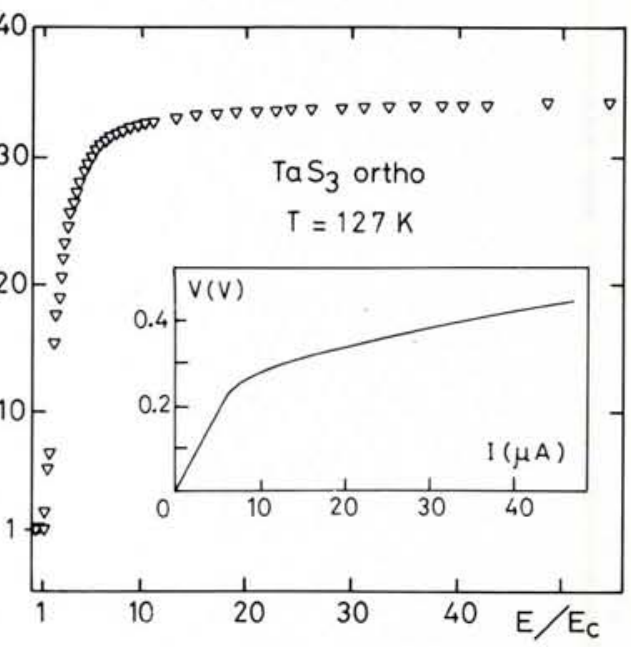

tends to the value $\sigma_{a}+\sigma_{b}$ which is of the order of the metallic conductivity extrapolated from above the critical temperature. $E_{\mathrm{T}}$ is also observed to increase significantly when the crystals are doped with impurities.

The low-field AC conductivity shows a strong increase in the range of 100 $\mathrm{MHz}-1 \mathrm{GHz}$ and then saturates at a value close to the DC infinite field limit. This behaviour can be described in terms of a harmonic oscillator response of the pinned CDW mode. For $\mathrm{NbSe}_{3}$ and $\mathrm{TaS}_{3}$ the response is overdamped. Recent measurements in the range of 10-100 $\mathrm{GHz}$ have revealed an inertial term that has led to an estimation being made of the CDW effective mass, the pinning frequency and the damping constant. However at low temperatures the single oscillator description fails and a distribution of pinning frequencies must be taken into account.

When $E$ is above $E_{T}$, a time-dependent voltage is generated in the crystal which can be studied with a spectrum analyser. Besides a broadband noise with a $1 / f$ frequency dependence, Fourier-transformed voltage spectra such as that shown in Fig. 3 for $\mathrm{NbSe}_{3}$, reveal a fundamental frequency and many harmonics. The fundamental frequency appears at $E_{\mathrm{T}}$ and increases with the current applied to the sample.

Steps can be observed in the DC V(I) characteristics if an RF current is superposed on a DC current exceeding $I_{\mathrm{T}}$. Such a synchronization effect is expected in non-linear oscillators and has in fact been observed in Josephson junctions (Shapiro steps) for frequencies in the immediate vicinity of the harmonic or subharmonics of the DC-current driven fundamental frequency. It is to be noticed that during synchronization, the sample nearly recovers its ohmic DC differential conductivity implying that near coherent oscillations are occurring over the whole sample. 
Because of the strong interaction of the CDW with impurities, it is unlikely that the CDW can be described by a unique ground state. Rather as in glasses, many metastable states have to be taken into account. Deformation of the CDW phase can be induced by a current and by temperature. The time scale for metastable states to relax to lower lying ones can be very broad depending on the material and the temperature; it is found that this decay time increases strongly at low temperature.

\section{Current Models for CDW Transport}

Bardeen ${ }^{7}$ ) was the first to interpret the non-linear conductivity in the materials described above as the Fröhlich conduction induced by the CDW motion. The major part of the theoretical work has been carried out for the incommensurate case, considering the extra conductivity as coming from a collective motion of the CDW phase.

The existence of a threshold field, $E_{\mathrm{T}}$, creates a problem as it can only be explained if we allow for a finite static deformation of the phase. Physically, a random distribution of impurities or dislocations is the most probable cause of the pinning force, but if we consider a completely rigid lattice, summation over individual pinning forces will give a square
Fig. 3 - Fourier transformed voltage spectra as a function of frequency for a $\mathrm{NbSe}_{3}$ sample (T $=42 \mathrm{~K}$ ) in the nonlinear state.

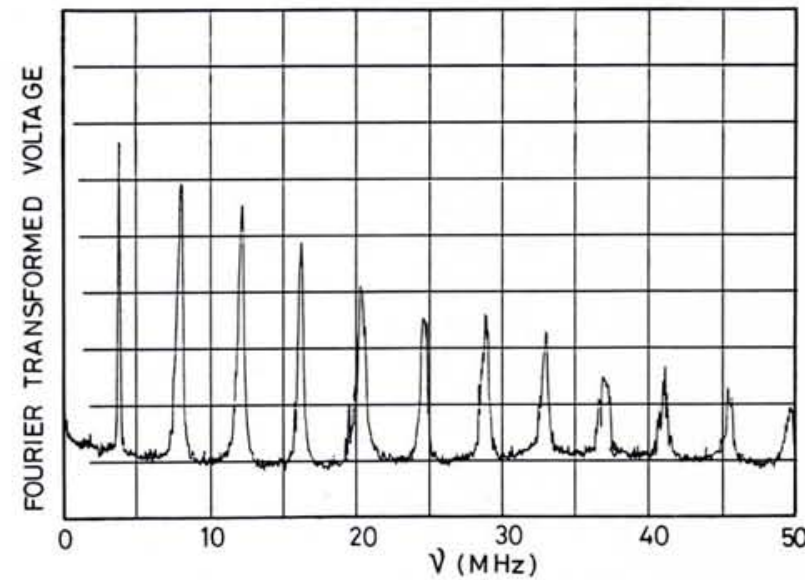

root dependence on the impurity content with $E_{\mathrm{T}}$ going to zero in the thermodynamic limit. In contrast, some elasticity allows for a deformation of the phase, and a finite second order effect.

A very popular model by Lee and Rice $\left.{ }^{8}\right)$ shows that the phase coherence between distant points tends to zero with distance if an arbitrarily small elasticity is introduced together with random pinning centres owing to the accumulation of small phase disturbances. They defined a "domain size" such that the phase deviation from the ideal is of the order of $\pi$, but in actual samples, domains are only a few microns across and any measurement is concerned with many domains. If it can be assumed that the domains act independently, then the total pinning force will be proportional to the sample length and $E_{\mathrm{T}}$ will be proportional to the square of the impurity concentration, and independent of length. It is far from clear what the thermodynamic limit of $E_{\mathrm{T}}$ means, but since experiments give finite values for relatively macroscopic samples, theories that consider domain motion have been developed.

A coherent domain is characterized by an equivalent mass $M$, some dissipative mechanism (thermalization of the phase

\section{Professor in Computational Solid State Physics}

\section{and leader of the Group "Electronic Structure of Materials" (ESM)}

The successful candidate will be leader of a group with a strong tradition in studies of the Electronic Structure of Materials (including band structure calculations) in relation to experimental results. He or she should be capable of continuing the extensive - national and international cooperation and should interact both with experimentalists and with theoretists in related fields.

The extraordinary professorship leads to a full-time appointment jointly financed by the Dutch National Science Foundation (FOM) and the Catholic University of Nijmegen (KUN). The group ESM which forms part of the interdisciplinary Research Institute of Materials (RIM) of the Science faculty also represents a joint venture of FOM and KUN.

The candidate should have a strong and wide background in solid state physics and modern computational methods. He should actively participate in studies of the relationship between the electronic structure of solids and their experimental properties through the calculation of these properties.

The candidate is expected to fulfill normal teaching duties (introductory general courses in physics and specialized courses in solid state physics) within the department of Physics - coordinated by the RIM - and the administrative obligations of a chair-holder and group leader.

Please send Curriculum Vitae including a list of publications and names of references to:

ms. drs. C.M. van den Heuvel (committee secretary), Algemeen Stafbureau

Katholieke Universiteit, Toernooiveld, 6525 ED Nijmegen, The Netherlands. Those wishing to draw attention to a potential candidate are welcome to contact the committee. 
motion by the phonon bath), a net charge $Q$, and an effective pinning force which must be periodic in $\phi$, since if $\phi$ is increased by $2 \pi$, each impurity sees the same charge distribution in its vicinity. This leads to an equation of motion

$$
M \ddot{\phi}+\Gamma \dot{\phi}+F_{0} \sin \phi=Q E
$$

This simple equation found in other physical contexts, e.g. Josephson contacts, dislocation dynamics, leads to at least qualitative explanations of many CDW phenomena:

- A threshold field defined by

$$
E_{\mathrm{T}}=F_{0} / Q
$$

- If $E=E_{0} \cos \omega t$ and $E_{0}<<E_{T}$, linearization of the sine term leads to a good agreement with the complex conductivity $\sigma(\omega)$ measured in low fields (linear response of an overdamped oscillator).

For a $\mathrm{DC}$ field $E>E_{\mathrm{T}}$, the sine term gives rise to a velocity modulation at a fundamental frequency, $v$, the harmonics of which can be considered as the origin of the periodic $A C$ voltage generated in these systems. It has to be noted that the assumed periodicity for the force, connected with the CDW wavelength, $\lambda_{\mathrm{CDW}}$ implies that the fundamental frequency is linked to the mean CDW velocity by

$$
v_{\text {CDW }}=\lambda_{\text {CDW }} v
$$

according to Eq. 3 the extra current density carried by the CDW in motion is therefore given by:

$$
J_{\mathrm{CDW}}=n_{0} e v_{\mathrm{CDW}}=n_{0} e \lambda_{\mathrm{CDW}} v
$$

A consequence of the classical equation of motion (Eq. 5) is that for $E$ slightly higher than $E_{\mathrm{T}}$, the extra DC current varies as:

$$
J_{\text {CDW }} \sim\left(E-E_{T}\right)^{1 / 2}
$$

However, experimental results show a nearly $3 / 2$ power law as shown in Fig. 4 , where the variation of the fundamental frequency is drawn for an orthorhombic $\mathrm{TaS}_{3}$ sample as a function of $\left(E-E_{\mathrm{T}}\right)$ at different temperatures. Attempts have been made to explain the regime near $E_{\mathrm{T}}$ by establishing some analogy between the vicinity of $E_{\mathrm{T}}$ and the critical behaviour at a second order phase transition, leading to the $3 / 2$ exponent.

According to Eq. 7 the slope of $J_{\mathrm{CDW}} / v$ is a measure of the number of electrons condensed below the CDW gap. The extra-current $J_{C D W}$ is measured from the non linear $V(I)$ characteristics. When $v$ is plotted as a function of $J_{\mathrm{CDW}}$, all the curves drawn in Fig. 4 collapse in a unique straight line (except for temperatures near the Peierls transition temperature). Fig. 5 shows the linear relationship between $J_{\mathrm{CDW}}$ and $v$ for an orthorhombic sample which is still valid with a CDW current density of $30000 \mathrm{~A} / \mathrm{cm}^{2}$. The number of electrons deduced from

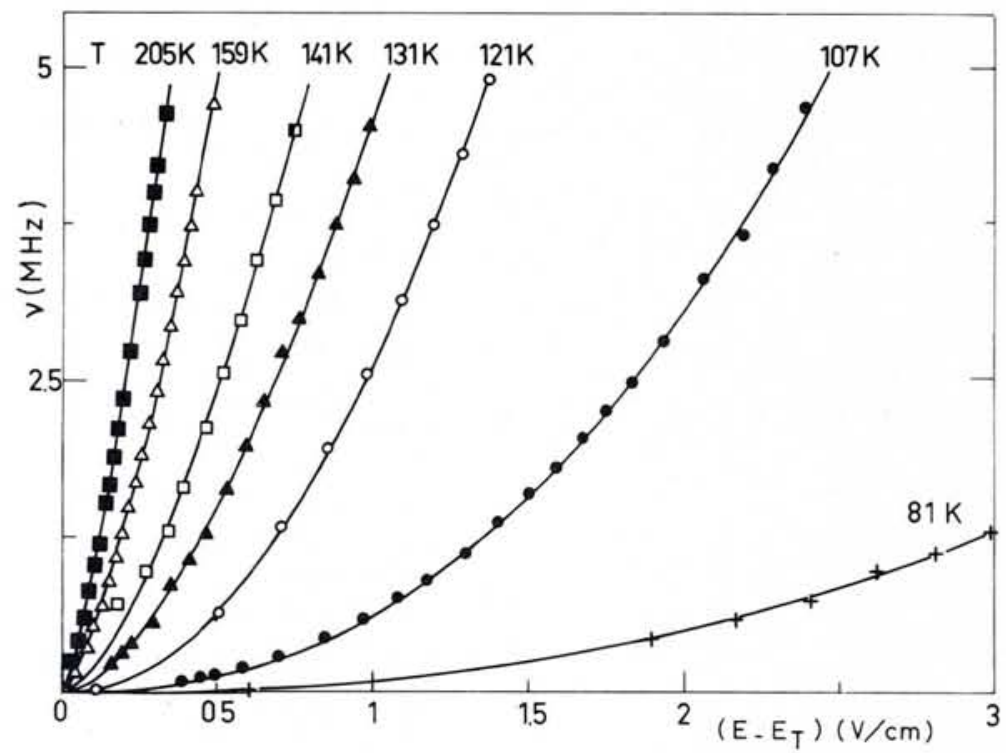

Fig. 4 - Variation of the fundamental noise frequency, $v$, measured in the Fourier-transformed voltage as a function of E-E $E_{T}$ at different temperatures for an orthorhombic $\mathrm{TaS}_{3}$ sample. The Peierls transition temperature is $T_{p}=215 \mathrm{~K}$. The lines are fits to the expression $\left(E-E_{T}\right)^{\gamma}$ with $\gamma \cong 1.5$.

the $v / J_{\text {CDW }}$ slope is, for any CDW compound, of the order of the electron concentration in the bands affected by the CDW condensation as can be calculated from band structures or from chemical bonds. This result is thought to be the proof of the Fröhlich conductivity: when the field overcomes the threshold, the electrons, which were trapped below the CDW gap, coherently participate in the electrical conductivity.

A quantum approach to the same problem has been put forward by Bardeen 7) and an alternative explanation has also been suggested: the motion of a discommensurate lattice. Discommensurations are thought of as bearing a charge so that their collective motion will carry a current.

Another controversial subject is the mechanism ensuring the condensation of electrons that must take place in the vicinity of electrical contacts supplying the DC current required to drive the CDW motion. Here also analogies with

vortex motion and Josephson effects in superconductors have provided useful insights.

\section{Conclusions}

Describing one-dimensional systems was for a long time of interest to theoreticians only, but in the 70's inorganic as well as organic chemists succeded in growing families of compounds relevant to this area of physics. Non-linear properties associated with the Peierls transition were also considered a mere curiosity so long as $\mathrm{NbSe}_{3}$ was the only material to exhibit such properties. Now, because of the availability of other compounds, it can be said that this behaviour is in fact a general property of pseudo one-dimensional systems as described above.

The sliding of the CDW nearly explains every feature at least qualitatively. However some problems remain to be solved. Among them is the increase of the threshold field at low temperature:

Fig. 5 - Variation of the current $J_{\text {CDW }}$ carried by the CDW as a function of the fundamental frequency measured in the Fourier-transformed voltage for an orthorhombic $\mathrm{TaS}_{3}$ sample at $T=127$ $\kappa$. The slope $J_{\text {Cow }} / v=$ ne $\lambda_{C D W}$ leads to the number of electrons condensed below the CDW gap.

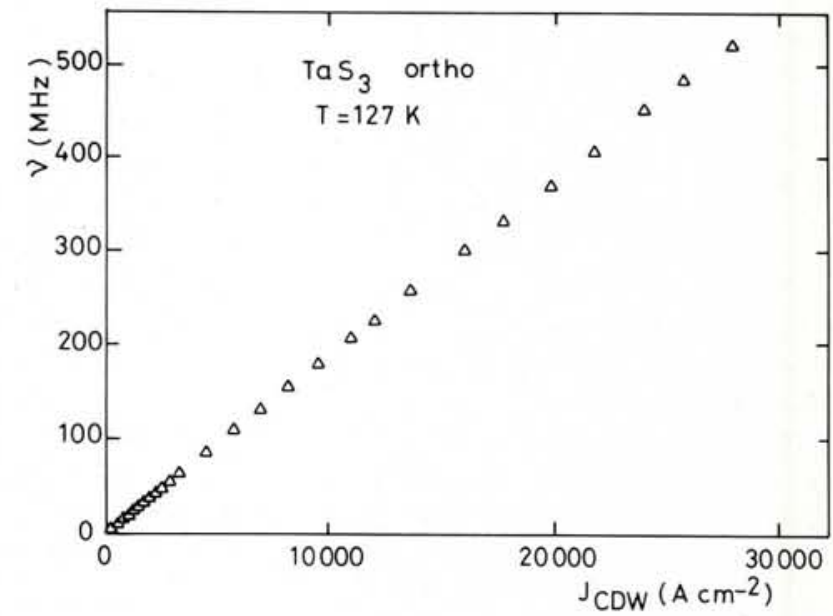


the only obvious energy scale is the gap so one would expect that the low temperature regime would be reached a few degrees below the Peierls transition temperature, yet as shown in Fig. 4 the apparent viscosity of the CDW increases strongly as the temperature is lowered. Further, the mechanism for the CDW damping and its temperature dependence remain to be clarified.

Definitive evidence for the sliding CDW would be a direct observation of its velocity. Recent NMR measurements ${ }^{9}$ ) show a motional narrowing of the NMR lineshape when the CDW is activated, which gives microscopic proof of the motion, but further experiments with the help of the tunnelling microscope could be even more convincing.

\section{BIBLIOGRAPHY}

1. Peierls R.F., Quantum Theory of Solids (Oxford University Press) 1955, p. 108.

2. Coleman R.V., Drake B., Hansma P.K. and Slough G., Phys. Rev. Lett. 55 (1985) 394.

3. Fröhlich H., Proc. Royal Soc. A 223 (1954) 296.

4. Lee P.A., Rice T.M. and Anderson P.W., Solid State Commun. 14 (1974) 703.

5. For a review see Proceedings of the International Conference on Charge Density Waves in Solids, held in Budapest in August 84, Lecture Notes in Physics Vol. 217, eds. Gy Hutiray and J. Solyom (Springer-Verlag, Berlin) 1985; Electronic Properties of Inorganic Quasi One-Dimensional Compounds Parts I and II, ed. P. Monceau (D. Reidel, Dordrecht) 1985 ;Crystal Chemistry and Properties of Materials with Quasi One-Dimensional Structures, ed. J. Rouxel (Reidel, Dordrecht) 1986.

6. Monceau P., Ong N.P., Portis A., Meerschaut A. and Rouxel J., Phys. Rev. Lett. 37 (1976) 602.

7. Bardeen J., Phys. Rev. Lett. 42 (1979) 1498 and 45 (1980) 1978.

8. Lee P.A. and Rice T.M., Phys. Rev. B 19 (1979) 3970.

9. Ross J.H. Jr., Wang Z. and Slichter C.P., Phys. Rev. Lett. 56 (1986) 663.

- Segransan P., Janossy A., Berthier C., Marcus J. and Butaud P., to be published.

\section{Meetings Issue}

The October issue of Europhysics News will comprise a calendar of meetings and schools of interests to physicists in Europe starting from January 1987. To guard against errors, organisers are requested to send their notices to the EPS Secretariat by 19 Sept. 1986 at the latest. All entries are included free of charge.

Organisers who believe that their event is eligible for EPS sponsorship should ask for an application form from the Secretariat and return it completed as soon as possible.

\section{The Pennsylvania State University Experimental Surface Physics}

The Department of Physics and the Materials Research Laboratory (MRL) are seeking candidates for a tenure-track faculty position in Experimental Surface Physics. Special preference will be given to individuals with experience in or related to scanning tunnelling microscopy techniques. Candidates should have a Ph.D. in Physics, an established record of research accomplishments and expect to set up a research program to complement the active surface physics program which exists in the Department. This appointment will be made jointly between the Department and MRL, allowing the full utilization of existing technologies in both units and the application of results to important materials problems. A desire and aptitude for teaching of undergraduate and graduate students is essential.

Send applications, including a curriculum vitae and names of at least four references, to

Professor Gerald A. Smith, Head, Department of Physics, Box S, The Pennsylvania State University, University Park, PA 16802

by October 15, 1986, or until a suitable pool of applicants is identified.

An affirmative action/equal opportunity employer.

\section{Computational Scientists Solid State Physics and Surface Science}

The Theory and Computational Science Division of the SERC's Daresbury Laboratory has two vacancies for work in computational solid state and surface physics.

The Laboratory is located in rural North Cheshire, and provides major facilities for scientific research by university groups. The present experimental facilities are centred on a $2 \mathrm{GeV}$ Synchrotron Radiation Source, and a $20 \mathrm{MV}$ van de Graaff accelerator. Computing facilities include an AS-7000 and FPS-164 attached processor on site, with access to the Cray 1-S in London, the CDC Cyber-205 in Manchester and a Cray X-MP at the Atlas Centre from early 1987.

These posts are to support the development of theoretical and computational methods for the Collaborative Computational Projects on Band Structure Theory (CCP9) and Surface Science (CCP3). These projects involve large and active groups of university collaborators with whom the successful candidates will interact. The major work of CCP9 over the next few years will be on highly accurate self-consistent methods for calculating the electronic structure of solids; the interests of CCP3 will centre on electronic properties of surfaces and atom-surface interactions. There is other work in progress in the Theory and Computational Science Division on solid state and surface physics, atomic and molecular physics, quantum chemistry, molecular dynamics and computer simulation of solids.

The successful candidates will have recently obtained a Ph.D. or expect to obtain one before taking up the appointment, preferably in theoretical or computational condensed matter physics. An appointment will be made at a salary (under review) between $f 7701$ and $f 11781$ according to age, ability and experience. The post will be available for a fixed term of three years and will be superannuable. There is flexibility in the starting date.

\section{CLOSING DATE: 15 September 1986}

Further information may be obtained from

Dr. J.E. Inglesfield (Tel. Warrington (925) 6031 21);

Dr. B.L. Gyorffy for CCP9 (University of Bristol, (272) 303030 Ext. 3677) or Professor J.B. Pendry FRS for CCP3 (Imperial College London, (1) 5895111 Ext. 6901).

Application forms may be obtained from:

The Personnel Officer, Daresbury Laboratory, Warrington WA4 4AD, Cheshire. Ref: DL/963: CCP3; DL/964: CCP9 Tel. (925) 603467 